\title{
Adaptive Approach for Modelling Variability in Pharmacokinetics
}

\author{
Andrea Y. Weiße $\mathrm{e}^{1,2}$, Illia Horenko ${ }^{2}$ and Wilhelm Huisinga ${ }^{1,2}$ \\ 1 DFG Research Center Matheon, and Department of Mathematics \\ 2 Informatics, Free University Berlin, Arnimallee 6, 14195 Berlin, Germany
}

\begin{abstract}
We present an improved adaptive approach for studying systems of ODEs affected by parameter variability and state space uncertainty. Our approach is based on a reformulation of the ODE problem as a transport problem of a probability density describing the evolution of the ensemble of systems in time. The resulting multidimensional problem is solved by representing the probability density w.r.t. an adaptively chosen Galerkin ansatz space of Gaussian distributions. Due to our improvements in adaptivity control, we substantially improved the overall performance of the original algorithm and moreover inherited the theoretical property that the number of Gaussian distribution stays constant for linear ODEs to the numerical scheme. We illustrate the approach in application to dynamical systems describing the pharmacokinetics of drugs and xenobiotics, where variability in physiological parameters is important to be considered.
\end{abstract}

\section{Introduction}

The medical benefits of a drug depend not only on its biological effect at the target protein, but also on its "life cycle" within the organism - from its absorption into the blood, distribution to tissue and its eventual breakdown or excretion by the liver and kidneys. Pharmacokinetics is the study of the drug-organism interaction, in particular the investigation of absorption, distribution, metabolism, and excretion (ADME) processes [9]. Studying ADME profiles is widely used in drug discovery to understand the properties necessary to convert leads into good medicines $[15,3]$. Physiologically based pharmacokinetic (PBPK) models aim at describing pharmacokinetic processes on a mechanistic basis. They model the body as a network of organ or tissue compartments that are interconnected by blood flow (see Fig. 1). From a mathematical point of view, a PBPK model comprises a system of coupled ordinary differential equations (ODEs). These equations involve physiological and physicochemical parameters, each of which is typically affected with uncertainty and some degree of variability due to interand intra-individual variations.

There are available theoretical and numerical tools for investigating ODEs with initial values and/or parameter uncertainty distributions. One class of approaches is represented by Monte Carlo methods based on a sampling of the initial distribution and subsequent solution of the underlying ODE for each of 


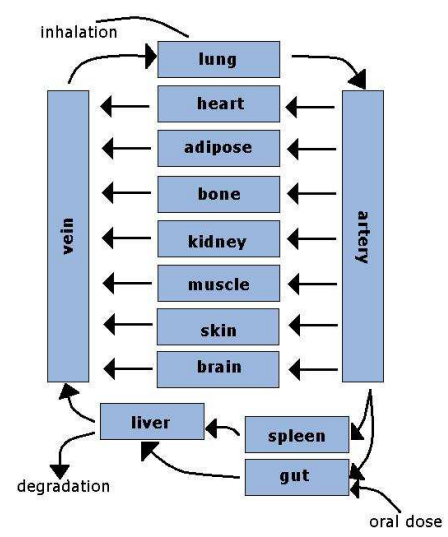

Fig. 1. A typical PBPK model. We distinguish between physiological and physicochemical parameters. Physiological parameters comprise organ volumes and blood flows; physicochemical parameters such as partition coefficients or solubility describe properties of the compound.

the sampling points (e.g. [14]). While this is the method of choice for problems with many parameters and degrees of freedom in order to avoid the "curse of dimensionality", the questions of numerical accuracy, reliability, and adaptivity still remain partially unclear. A second class of methods are sparse grid techniques $[16,4]$ or particle methods $[13,11]$. In contrast to frequently used conventional grid methods, they both scale reasonably well for medium dimensional problems. Sparse grids work best for smooth anisotropic densities with the grid being aligned to the propagated objects. Adaptivity is used to generate an optimal sparse grid in order to minimize the approximation error beneath some predefined threshold. However, for isotropic problems, e.g., for classical Liouville or Fokker-Planck equations with Gaussian initial density, the adaptive sparse grid methods end up by full grids, i.e., they are practically not applicable for high-dimensional isotropic problems. A third class of approaches is known as the stochastic finite elements (SFEMs) approach [7,8,12]. This method represents the overall statistical response of the system by a linear combination of orthogonal basis functions. However, in the available form, this approach cannot be applied to higher dimensional problems with different time and length scales as it is typical for reaction kinetics and pharmacokinetic models.

In this article, we present a theoretical framework and an improved adaptive numerical approach for systems of ODEs affected by parameter variability and uncertainty distributions. The problem is reformulated in terms of the well-established Frobenius-Perron theory, involving the semigroup of FrobeniusPerron operators. In order to approximate the semi-group numerically, we adopt and substantially extend the adaptive Gaussian-based particle method TRAIL [6] that has originally been developed in the context of molecular dynamics [6] and recently be transferred to reaction systems [5]. The approach is based on two ingredients, (i) a time-dependent Galerkin ansatz space of Gaussian basis 
functions, and (ii) a propagation of the density w.r.t. the Galerkin ansatz space. First applications to reaction kinetics demonstrated the potential of the method, however the adaptivity control remained unsatisfactory from both the theoretical as well as the efficiency point of view. In this article we propose an improved adaptivity control and demonstrate its power in application to typical systems in pharmacokinetics, where variability and uncertainty play an important role.

\section{Theoretical background}

Let us assume that the dynamics is defined in terms of some ODE

$$
\dot{x}=f(x \mid p)
$$

with some continuously differentiable right hand side $f(\cdot \mid p): \mathbf{X} \rightarrow \mathbb{R}^{d}$ parameterized by $p \in \mathbf{Y} \subset \mathbb{R}^{m}$, the vector of fixed parameters. In the setting of pharmacokinetics, $\mathbf{X} \subset \mathbb{R}^{d}$ is the space of concentrations in $d$ compartments, and $p$ represents physiological and physicochemical parameters. Given the initial state $x(0)=x_{0}$, the solution of the initial value problem $(1), x(t \mid p): \mathbb{R} \rightarrow \mathbf{X}$ describes the concentration-time behavior in $d$ different tissues and can formally be written in terms of the flow $\Phi^{t}(\cdot \mid p)$

$$
x(t \mid p)=\Phi^{t}\left(x_{0} \mid p\right)
$$

that is known to be invertible. Now assume that the parameters are specified in terms of some statistical distribution in contrast to a fixed numerical value. Rather than solving (1) for some initial value $x_{0}$ and a single set of parameters, we are interested in capturing the effects of distributed parameters on the evolution of the dynamics, i.e, the initial value. Then, eq. (1) becomes an ODE with random parameters and possibly random initial conditions that can be interpreted as the evolution of an entire population. We can easily extend equation (1) to account for influences of distributed parameters by extending the state space to $\mathbf{Z}:=\mathbf{X} \times \mathbf{Y}$ and setting $\dot{p}$ to zero

$$
\left(\begin{array}{c}
\dot{x} \\
\dot{p}
\end{array}\right)=F(x, p)=\left(\begin{array}{c}
f(x \mid p) \\
0
\end{array}\right)
$$

since parameters are assumed to be constant in time.

Denote by $\mathcal{L}^{2}(\mathrm{~d} x \mathrm{~d} p)$ the space of square integrable functions. The semigroup of Frobenius-Perron operators $P_{t}: \mathcal{L}^{2}(\mathrm{~d} x \mathrm{~d} p) \rightarrow \mathcal{L}^{2}(\mathrm{~d} x \mathrm{~d} p)$ associated with $(3)$ describes the evolution of a given density $u_{0} \in \mathcal{L}^{2}(\mathrm{~d} x \mathrm{~d} p)$ in time according to $P_{t} u_{0}=u_{t}$. Since the flow $\Phi^{t}$ is invertible and differentiable, $P_{t}$ is explicitly defined by

$$
P_{t} u_{0}(x, p)=u_{0}\left(\Phi^{-t}(x, p)\right) \cdot\left|\frac{\mathrm{d} \Phi^{-t}(x, p)}{\mathrm{d} x \mathrm{~d} p}\right|
$$

where the last term denotes the determinant of the Jacobian of $\Phi^{-t}[10]$. In broad terms, definition (4) can be interpreted as follows: the value of the density $u_{t}$ at 
$(x, p)$ is given by the value of $u_{0}$ at the pre-image of $(x, p)$ corrected according to the dynamics (expanding or contracting directions). The infinitesimal generator $\mathcal{A}$ of the semigroup of Frobenius-Perron operators is defined by

$$
\mathcal{A} u=-\operatorname{div}(F \cdot u)=-\sum_{i=1}^{n} \frac{\partial}{\partial z_{i}}(F \cdot u),
$$

where $z \in \mathbf{Z}$ and $n=d+m$ is the dimension of $\mathbf{Z}$.

\section{Adaptive density propagation}

For the density propagation of Liouville type problems, Horenko and Weiser developed a multidimensional, adaptive particle method to describe the propagation of distributions in non-linear dynamical systems, called TRAIL (trapezoidal rule for adaptive integration of Liouville dynamics) [6]. The adaptive discretization scheme is based on a semi-discretization in time and subsequent approximation of the stationary spatial problem. The key idea is to approximate the distribution $u$ w.r.t. a time-adapted Galerkin basis of Gaussian ansatz functions.

Semi-discretization in time. Consider a probability distribution $u_{0}$ characterizing variability and uncertainty of parameters and state variables. Then, at any later time $t>0$ the distribution $u_{t}$ is given by $u_{t}=P_{t} u_{0}$ involving the semi-group of Frobenius-Perron operators. The basic idea of temporal semi-discretization is to approximate $\left\{P_{t}\right\}_{t \geq 0}$ by a simpler and numerically treatable semi-group $\left\{R_{t}\right\}_{t \geq 0}$. Loosely speaking, since $P_{t}=\exp (t \mathcal{A})$, we define $R_{t}=r(t \mathcal{A})$ based on some possible rational approximation $r(\cdot)$ of the exponential function $\exp (\cdot)$. Relevant in our context are $R_{t}=(\mathrm{Id}-t \mathcal{A} / 2)^{-1}(\mathrm{Id}+t \mathcal{A} / 2)$ denoting the trapezoidal rule and $R_{t}=\mathrm{Id}+t \mathcal{A}$ denoting the explicit Euler scheme. Finally, the numerical scheme exploits the semi-group property to approximate $P_{t}$ for some large $t>0$ according to $P_{t}=P_{\tau_{n}} \circ \cdots \circ P_{\tau_{n}} \approx R_{\tau_{n}} \circ \cdots \circ R_{\tau_{n}}$ for some adaptively chosen sequence of time steps $t=\tau_{n}+\cdots+\tau_{1}$.

Spatial discretization of stationary problem. At the very beginning, the initial distribution $u_{0}$ is approximated by a finite sum of Gaussian distributions, i.e.,

$$
u_{0}=\sum_{j=1}^{N_{t_{0}}} \omega_{j}\left(t_{0}\right) B_{j}\left(\cdot ; t_{0}\right)+\delta_{t_{0}}
$$

such that $\left\|\delta_{t_{0}}\right\|<\mathrm{TOL}$, and for $j=1, \ldots, N_{t}$ and $t \geq 0$

$$
B_{j}(z ; t):=\exp \left\{\left(z-\mu_{j}(t)\right)^{T} G_{j}(t)\left(z-\mu_{j}(t)\right)+a_{j}(t) .\right\}
$$

The parameters $\mu_{j}(t), G_{j}(t)$, and $a_{j}(t)$ with $j=1, \ldots, N_{t}$ denote the corresponding means, inverses of the covariance matrices and normalization constants ${ }^{3}$,

\footnotetext{
3 The constants are chosen in such a way that $B_{j}(\cdot ; t)$ is normalized to one, resulting in $a_{j}(t)=\ln \left(\operatorname{det}\left(2 \pi \Sigma_{j}(t)\right)^{-\frac{1}{2}}\right)$.
} 
respectively. For details, see $[6,5]$. The initial approximation also defines the Galerkin basis $\left\{B_{j}\left(\cdot ; t_{0}\right): j=1, \ldots, N_{t_{0}}\right\}$ at time $t_{0}$.

In each step, the scheme comprises two steps: (i) adaptation of the Galerkin basis w.r.t. the underlying dynamics; (ii) optimal representation of the timepropagated density w.r.t. the adapted Galerkin basis. The propagation of the Galerkin basis is performed w.r.t. to the locally (around each mean $\mu_{j}$ ) linearized dynamics guaranteeing that the Gaussian distributions remain Gaussian in time. The parameters evolve according to the ODEs:

$$
\begin{aligned}
\dot{a_{j}} & =-\operatorname{trace}\left(D F\left(\mu_{j}\right)\right) \\
\dot{\mu}_{j} & =F\left(\mu_{j}\right) \\
\dot{G}_{j} & =-D F\left(\mu_{j}\right)^{T} G_{j}-G_{j} D F\left(\mu_{j}\right),
\end{aligned}
$$

where $D F(z)$ denotes the Jacobian of $F$ at $z \in \mathbf{Z}$. The representation of the timepropagated density w.r.t. to the new Galerkin basis is realized via the trapezoidal rule. The new coefficients $\left\{\omega_{j}(t+\tau): j=1, \ldots, N_{t}\right\}$ are optimized according to

$$
\left\|\delta_{t+\tau}\right\|=\left\|\left(1-\frac{\tau}{2} \mathcal{A}\right) u(\cdot, t+\tau)-\left(1+\frac{\tau}{2} \mathcal{A}\right) u(\cdot, t)\right\|=\min
$$

with

$$
u(\cdot, t+\tau)=\sum_{j=1}^{N_{t}} \omega_{j}(t+\tau) B_{j}(\cdot ; t+\tau) .
$$

This can efficiently be performed by approximating the involved norm by a Monte-Carlo sampling, reformulating eq. (11) as a least squares problem to be solved by means of a qr-algorithm [6], and noting that the action of the infinitesimal generator $\mathcal{A}$ on a Gaussian basis function can be computed exactly at any given state $z \in \mathbf{Z}$.

Adaptivity in time and space. The crucial ingredient of the TRAIL scheme is the adaptive choice of the next time step and the adaptation of the Galerkin basis (increasing or decreasing the number of basis functions) to keep the local error below a user-defined local tolerance TOL. In the original TRAIL scheme, temporal adaptivity is realized by a step size control based on a comparison of the trapezoidal rule and the Euler scheme, while spatial adaptivity is realized by exploiting properties of the qr-algorithm in the optimization step combined with some "accuracy matching" (splitting of the local tolerance into some temporal and spatial local tolerance), for details, see [6].

\section{Improved adaptivity}

It is well-known that for linear ODEs an initially Gaussian distribution stays Gaussian in time. In terms of the TRAIL scheme this means that the number of Gaussian basis functions should stay constant for linear ODEs. However, 
application to simple linear ODEs revealed that the number of Gaussians basis functions does in general not stay constant unless an a-priori unknown small maximal time step is introduced. Since the numerical effort of the TRAIL scheme scales cubically with the number of Gaussian basis functions, this result is unsatisfactory from both a numerical (adaptivity and efficiency) point of view as well as from a theoretical point of view. Rather one would like to design an adaptivity control that allows for efficiency and inherits the theoretical properties for linear ODEs to the numerical scheme. A thorough analysis of the performance for linear problems revealed that new ansatz functions are added due to a too coarse time discretization resulting in an overestimation of the spatial error. At the same time, conservation of (probability) mass is poor. On the other hand, bounding the time-step from above by some maximal time step (a-priori unknown and in general depending on the ODE and the initial distribution) enforced a constant number of ansatz functions, but slowed down the integration drastically.

The improved adaptivity control is based on the key idea to control the time-step not only based on a comparison of two different numerical schemes (in the case of TRAIL the trapezoidal rule and the Euler scheme), but also based on the spatial discretization error (estimator): For linear ODEs the timestep is rejected and subsequently decreased whenever the spatial error estimator exceeds the spatial tolerance. In addition, we replace the Euler method by a second order Runge-Kutta methods with $R_{t}=\mathrm{Id}+t \mathcal{A}+t^{2} \mathcal{A}^{2} / 2$ for a more efficient performance. For non-linear ODEs, the same is done based on a (local) linearization of the ODE. Hence in broad terms, the time step is controlled in such a way that a change in the number of basis functions is only due to nonlinear effects of the underlying dynamics. In the next section we demonstrate the improved adaptivity control in application to two typical pharamcokinetics models.

\section{Numerical examples}

This section illustrates the numerical scheme in application to two model problems in pharmacokinetics.

The first model is a very simple empirical two-compartment model that is frequently used in population pharmacokinetics to analyze large data sets resulting from clinical studies. It comprises two compartments, a central and a peripheral one. Typically, the central compartment is thought of as blood compartment, while the peripheral compartment is empirically chosen. In contrast to physiologically based models this type of model is empirical. A compound is transferred from the central to the peripheral compartment with some transfer rate $k_{t}$, where it is eliminated with some elimination rate $k_{e}$. The resulting ODEs are:

$$
\left(\begin{array}{c}
\dot{C}_{c} \\
\dot{C}_{p}
\end{array}\right)=\left(\begin{array}{cc}
-k_{t} & 0 \\
k_{t} & -k_{e}
\end{array}\right)\left(\begin{array}{l}
C_{c} \\
C_{p}
\end{array}\right)
$$

where $C_{c}$ and $C_{p}$ denote the concentrations of the compound in the central and peripheral compartment. The evolution of the system with for initially distri- 
bution concentrations is depicted in Fig. 2. In the left column, fixed parameter values are chosen: $k_{t}=0.5$ and $k_{e}=0.6$, while in the right column parameter variability according to $k_{t} \sim \mathcal{N}(0.5,0.01)$ and $k_{e} \sim \mathcal{N}(0.6,0.01)$ is taken into account.

Though variances of $k_{t}$ and $k_{e}$ are comparatively small, we observe considerable effects on the joint distribution of central and peripheral concentrations. By extending the state space, the ODE has become non-linear. Still, the number of ansatz functions remains constant for the simulation time performed. This might indicate that non-linear effects have not been dominated. Applying the original TRAIL scheme without the presented improvements results in a large number of Gaussian ansatz functions ( $>100$ depending on the user prescribed tolerance) for both the linear model (only concentrations) as well as the non-linear model (concentration and parameters).
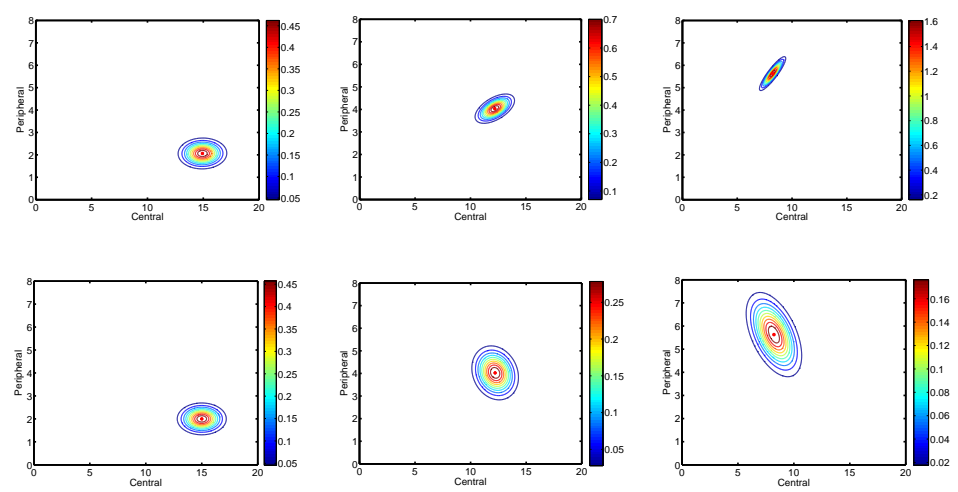

Fig. 2. Simulations of system (12) with initial concentrations $C_{c}(0) \sim \mathcal{N}(15,1)$ and $C_{e}(0) \sim \mathcal{N}(2,0.1)$. The left column shows the evolution of the initial density without parameter variation, whereas in the right column the state space has been extended by $k_{t}$ and $k_{e}$. In both cases joint distributions of central and peripheral concentrations are shown for time points $t=0,0.4,1.2 \mathrm{~h}$ (bottom to top).

Next, we simulate and analyze a physiologically-based pharmacokinetic model that has been developed in the context of toxicological risk assessment $[?, 1,2]$. The PBPK model comprises the organs/tissues liver, adipose, muscle, and vessel rich tissue that are interconnected by the blood flow. Based on the law of mass action a system of coupled ODEs describing the time-evolution of the concentrations in the above organs and tissues is established. For org $\in\{$ liver, adipose, muscle, and vessel rich tissue we get

$$
V_{\text {org }} \frac{\mathrm{d} C_{\text {org }}}{\mathrm{d} t}=Q_{\text {org }} \cdot\left(C_{\text {art }}-\frac{C_{\text {org }}}{P_{\text {org }}}\right),
$$

where $V_{\text {org }}, Q_{\text {org }}$ and $P_{\text {org }}$ denote the volume, blood flow and the so-called tissue partition coefficient, respectively. In the liver compartment, additionally a non- 

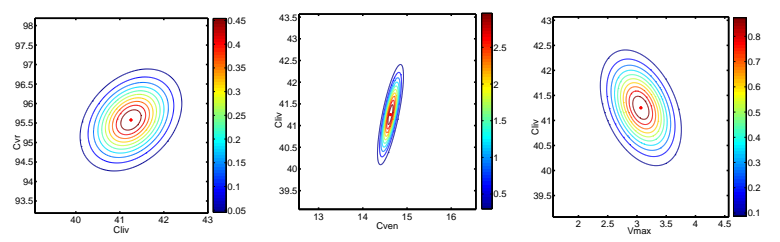

Fig. 3. Simulations of the ten dimensional extended styrene system. Two dimensional projections of the high dimensional joint distribution are shown for concentrations in liver \& vessel rich tissue (left), venous blood \& liver (center), and $V_{\max }$ with liver concentration (right) are shown.

linear Michaelis-Menten term accounting for saturable metabolism is present. The equations for venous and arterial blood are given by

$$
\begin{aligned}
V_{v e n} \frac{\mathrm{d} C_{v e n}}{\mathrm{~d} t} & =\sum_{i \in \text { Tissues }} Q_{i} \cdot \frac{C_{i}}{P_{i}}-C_{\text {ven }} \cdot Q_{t o t} \\
V_{\text {art }} \frac{\mathrm{d} C_{\text {art }}}{\mathrm{d} t} & \left.=Q_{t o t} \cdot\left(C_{\text {ven }}-C_{\text {art }}\right)+Q_{\text {alv }} \cdot\left(C_{\text {inh }}-\frac{C_{\text {art }}}{P_{\text {air }}}\right)\right),
\end{aligned}
$$

where $C_{i n h}$ denotes styrene concentration in inhaled air, $Q_{a l v}$ alveolar flow, and $Q_{t o t}$ the total blood flow.

In [2], it was found that the system is sensitive to parameter uncertainty w.r.t. $V_{\max }$ and $K_{m}$ in liver tissue, the blood:air partition coefficient $P_{a i r}$, and the partition coefficient in adipose tissue $P_{f a t}$. Hence, in addition to the ODE for the concentrations we take uncertainty of these parameters into account. The simulation results for the ten dimensional PBPK system modelling the inhalation of styrene in human body are shown in Fig. 3.

The initially uncorrelated distributions develop correlations in time, both between different tissues (Fig. 3, left and center) and between liver concentration and $V_{\max }$ (Fig. 3, right). As we would expect, liver concentration is negatively correlated to $V_{\max }$. Also we would expect a positive correlation for liver and venous concentrations, since both compartments are directly coupled by the liver blood flow. The positive correlation of vessel rich and liver tissue on the other hand is not obvious on first sight. Knowledge about the effect of variability and uncertainty is important information for risk assessment studies, as in [1].

\section{Conclusions}

We presented an improved approach for adaptive density propagation in the context of ODEs affected by parameter variability and state space uncertainty. Our approach is based on a representation of the corresponding probability density w.r.t. an adaptively chosen Galerkin ansatz space of Gaussian distributions. Due to our improvements in adaptivity control, the theoretical property that the number of Gaussian distribution stays constant for linear ODEs is now inherited to the numerical scheme. Since the numerical efforts scale with the third 
power of the number of Gaussian basis functions, we managed to substantially improve the overall performance. In addition the conservation of (probability) mass improved.

Most often, Monte Carlo (MC) approaches are applied to study dynamical systems with distributed parameters and states. The MC methods generate an ensemble of sampling points that approximate the statistical distribution. However, in contrast to molecular dynamics, where the underlying Hamiltonian structure implies conservation of phase volume and probability density along trajectories, this properties does rarely hold in reaction kinetics and pharmacokinetics. As a consequence, a single sampling point is of limited use and only in form of expectation values relevant information can be extracted. Moreover, the control of the approximation error still remains partially unclear. The adaptive approach to density propagation presented herein generates a continuous approximation of the density in time. Since the density is approximated in terms of Gaussian distributions, the approach is expected to be efficient whenever the underlying dynamics results in densities that are "sufficiently smooth", as it seems to be the case for pharmacokinetics problems. Due to the improved adaptivity control it might also become possible to selectively study the influence of non-linear effects on the overall dynamics by monitoring the dimension of the Galerkin basis, i.e., the number of basis functions, since only the non-linear part of the dynamics is able to increase or decrease the number of Gaussians. This would allow to extract a completely different and very interesting type of information and is currently under investigation.

The results in application to pharmacokinetic models demonstrate the advantages of the approach presented. As a result of the simulation studies, detailed information on the distribution in state space and, e.g, the correlation between different parameters is available. These are important data for toxicological risk assessments [1], or to study the variability of a drug exposure in an entire population (an information becoming more and more important).

\section{Acknowledgment}

A.Y.W. has been supported by the International Max Planck Research School for Computational Biology and Scientific Computing (IMPRS-CBSC), Berlin, and by the Konrad-Zuse-Zentrum für Informationstechnik Berlin (ZIB). I.H. acknowledges financial support by SFB 450. W.H. acknowledges financial support by the DFG Research Center MATHEON "Mathematics for key technologies", Berlin.

\section{References}

1. K. Abraham, H. Mielke, W. Huisinga, and U. Gundert-Remy. Elevated internal exposure of children in simulated acute inhalation of volatile organic compounds: effects of concentration and duration. Arch Toxicol, 79(2):63-73, Feb 2005. 
2. K. Abraham, H. Mielke, W. Huisinga, and U. Gundert-Remy. Internal exposure of children by simulated acute inhalation of volatile organic compounds: the influence of chemical properties on the child/adult concentration ratio. Basic Clin Pharmacol Toxicol, 96(3):242-243, Mar 2005.

3. A.P. Beresford, H.E. Selick, and Michael H. Tarbit. The emerging importance of predictive adme simulation in drug discovery. DDT, 7:109-116, 2002.

4. M. Griebel and G. W. Zumbusch. Adaptive sparse grids for hyperbolic conservation laws. In M. Fey and R. Jeltsch, editors, Hyperbolic Problems: Theory, Numerics, Applications. 7th International Conference in Zürich, February 1998, International Series of Numerical Mathematics 129, pages 411-422, Basel, 1999. Birkhäuser. http://wissrech.ins.uni-bonn.de/research/pub/zumbusch/hyp7.pdf.

5. I. Horenko, S. Lorenz, Ch. Schütte, and W. Huisinga. Adaptive approach for nonlinear sensitivity analysis of reaction kinetics. J. Comp. Chem., 26(9):941-948, 2005.

6. Illia Horenko and Martin Weiser. Adaptive integration of molecular dynamics. Journal of Computational Chemistry, 24(15):1921-1929, 2003.

7. Andreas Keese. A review of recent developments in the numerical solution of stochastic partial differential equations (stochastic finite elements). Informationbericht 2003-6, Department of Computer Science, Technical University Braunschweig, Brunswick, Germany, Institute of Scientific Computing, Technische Universität Braunschweig, Hans-Sommer-Straße 65, D-38106 Braunschweig, October 2002.

8. M. Kleiber and T. D. Hien. The stochastic finite element method, Basic perturbation technique and computer implementation. J. Wiley and Sons, 1992.

9. Younggil Kwon. Handbook of Essential Pharmacokinetics, Pharmacodynamics, and Drug metabolism for Industrial Scientists. Kluwer Academic/Plemun Publishers, 2001.

10. A. Lasota and M. C. Mackey. Chaos, Fractals, and Noise: Stochastic Aspects of Dynamics, 2nd Ed. Springer-Verlag, Berlin, 1995.

11. Marc A. Schweitzer M. Griebel. Meshfree Methods for Partial Differential Equations. Lecture Notes in Computational Science and Engineering. Springer, Berlin, Heidelberg, New York, 2003.

12. Hermann G. Matthies and Marcus Meyer. Nonlinear galerkin methods for the model reduction of nonlinear dynamical systems. Informationsberich 2002-3, Department of Computer Science, Technical University Braunschweig, Brunswick, Germany, March 2002. submitted to special issue of Computers and Structures, Revised and extended version of a contribution to the EUROMECH Colloquium, 427, ENS Cachan France, September 2001.

13. H. Neunzert, A. Klar, and J. Struckmeier. Particle methods: Theory and applications. In ICIAM 95: proceedings of the Third International Congress on Industrial and Applied Mathematics held in Hamburg, Germany, 1995.

14. D. Talay. Probabilistic numerical methods for partial differential equations: elements of analysis. In D. Talay and L. Tubaro, editors, Probabilistic Models for Nonlinear Partial Differential Equations, Lecture Notes in Mathematics 1627, pages 48-196. Springer, 1996.

15. H. van Waterbeemd and E. Gifford. Admet in silico modelling: towards prediction paradise? Nature, 2:192-204, 2003.

16. Christoph Zenger. Sparse grids. In Wolfgang Hackbusch, editor, Parallel Algorithms for Partial Differential Equations, volume 31 of Notes on Numerical Fluid Mechanics, pages 241-251. Vieweg, 1991. 